\title{
Toward automated cochlear implant insertion using tubular manipulators
}

Josephine Granna, Thomas S. Rau, Thien-Dang Nguyen, Thomas Lenarz, Omid Majdani, et al.

Josephine Granna, Thomas S. Rau, Thien-Dang Nguyen, Thomas Lenarz, Omid Majdani, Jessica Burgner-Kahrs, "Toward automated cochlear implant insertion using tubular manipulators," Proc. SPIE 9786, Medical Imaging 2016: Image-Guided Procedures, Robotic Interventions, and Modeling, 97861F (18 March 2016); doi: 10.1117/12.2216854

SPIE. Event: SPIE Medical Imaging, 2016, San Diego, California, United States 


\title{
Toward Automated Cochlear Implant Insertion using Tubular Manipulators
}

\author{
Josephine Granna ${ }^{a}$, Thomas S. Rau ${ }^{b}$, Thien-Dang Nguyen ${ }^{a}$, Thomas Lenarz ${ }^{b}$, \\ Omid Majdani ${ }^{b}$ and Jessica Burgner-Kahrs ${ }^{a}$ \\ ${ }^{a}$ Center of Mechatronics (MZH), Leibniz Universität Hannover, Hanover, Germany; \\ ${ }^{b}$ Department of Otolaryngology, Hannover Medical School (MHH), Hanover, Germany
}

\begin{abstract}
During manual cochlear implant electrode insertion the surgeon is at risk to damage the intracochlear finestructure, as the electrode array is inserted through a small opening in the cochlea blindly with little forcefeedback. This paper addresses a novel concept for cochlear electrode insertion using tubular manipulators to reduce risks of causing trauma during insertion and to automate the insertion process.

We propose a tubular manipulator incorporated into the electrode array composed of an inner wire within a tube, both elastic and helically shaped. It is our vision to use this manipulator to actuate the initially straight electrode array during insertion into the cochlea by actuation of the wire and tube, i.e. translation and slight axial rotation. In this paper, we evaluate the geometry of the human cochlea in 22 patient datasets in order to derive design requirements for the manipulator. We propose an optimization algorithm to automatically determine the tube set parameters (curvature, torsion, diameter, length) for an ideal final position within the cochlea. To prove our concept, we demonstrate that insertion can be realized in a follow-the-leader fashion for 19 out of 22 cochleas. This is possible with only 4 different tube/wire sets.
\end{abstract}

Keywords: tubular manipulators, cochlear implants, continuum robots, robot assisted surgery

\section{DESCRIPTION OF PURPOSE}

A cochlear implant (CI) is a neural prosthesis which restores lost hearing ability through electrical stimulation of the auditory nerve deployed for patients suffering from hearing loss. An electrode array composed of a silicone body with multiple electrodes is therefore inserted into the diminutive helical shaped cochlea (mean canal diameter below $2 \mathrm{~mm}$ ). Nowadays, improvements in surgical technique enable cochlear implant provision also for patients with substantial residual hearing, if the insertion of the electrode is done in an atraumatic manner and residual hearing is preserved. During the last decade CI manufactures developed new electrode arrays to address the challenge of hearing preservation. However, these implants have several drawbacks: on the one hand, there are still non-negligible implant-to-tissue interactions with remaining insertion forces and on the other hand, the electrode array is not finally placed close to the inner wall of the inner ear, also known as perimodiolar placement. Existing perimodiolar electrode arrays are, in contrast, not suitable for hearing preservation surgery due to their higher thickness and stiffness. Thus, it is still an unsolved problem to provide an electrode array which reliable combines both atraumatic insertion and perimodiolar placement. Different strategies use existing perimodiolar electrode arrays and optimize the shape and position with respect to the individual anatomy ${ }^{1}$ or equip electrode arrays with an active bending mechanism. ${ }^{23456}$

As continuum robotic research aims in developing manipulators, which can reach hardly accessible anatomical sites inside the human body, e.g. through natural orifices, we foresee tubular continuum robots to be predestined for cochlear implant surgery. Tubular continuum robots (also referred to as concentric tube robots) are the smallest continuum manipulators developed thus far, which exhibit a continuous structure and are composed of multiple precurved, elastic tubes nested inside one other. ${ }^{7}$ Actuation of the tubes is achieved mechanically by translating and rotating the base of each tube. Proposed medical applications using tubular continuum robots include for instance cardiac surgery, ${ }^{8}$ transurethral urologic surgery, ${ }^{9}$ transnasal skull base surgery, ${ }^{10}$ or intracerebral hemorrhage evacuation. ${ }^{11}$ If tubular manipulators should deploy along tortuous paths, follow-theleader motion is required. Follow-the-leader deployment means, that the manipulator's shape corresponds to the

Medical Imaging 2016: Image-Guided Procedures, Robotic Interventions, and Modeling, edited by Robert J. Webster III, Ziv R. Yaniv, Proc. of SPIE Vol. 9786, 97861F

(C) 2016 SPIE - CCC code: $1605-7422 / 16 / \$ 18 \cdot$ doi: $10.1117 / 12.2216854$ 
path of its tip at all times. This is not trivial to achieve with tubular manipulators as the elastic interaction between the tubes during actuation determines the shape. Tube parameters have to be selected wisely, as discussed in. ${ }^{12}$ This motivated our research toward utilization of tubular manipulators in cochlear implant electrode insertion, where a follow-the-leader behavior of tubular manipulators is essential.

We propose a tubular manipulator to realize a steerable electrode which adjusts its curling behavior to the individual shape of the inner ear. As a result, we expect an improved and more exact placement of the electrode array. It is our vision that tubular manipulators will substitute the stylet, a wire which straightens the electrode array during insertion and is commonly used nowadays for perimodiolar placement. Figure 1 illustrates the cochlea with its main two ducts: the lower scala tympani and upper scala vestibuli. A tubular manipulator is moving within a guide channel of the elec-

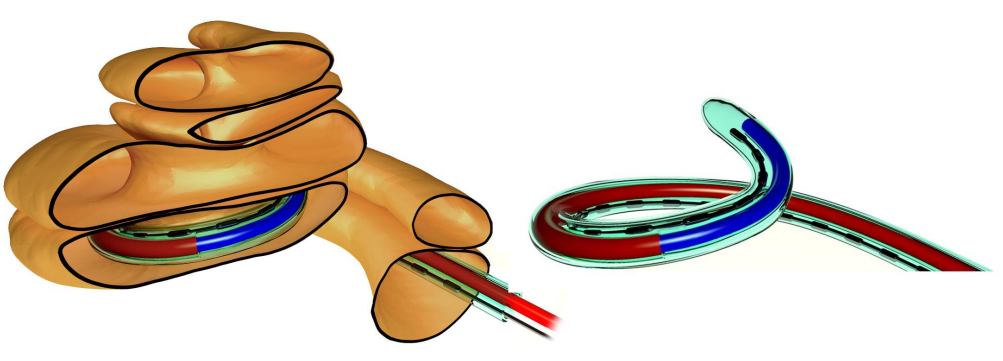

Figure 1: Envisioned steerable electrode array with an helical tube (red) and wire (blue) in a guide channel for insertion into the scala tympani (lower duct of cochlea). trode silicone body and thus replaces the stylet of common cochlea electrodes. The manipulator is composed of an outer tube and an inner wire, both elastic and preshaped such that their combined shape matches the course of the scala tympani. We envision an optimal electrode array placement within the scala tympani, by actuating the manipulator's tube and wire during electrode insertion. We have recently demonstrated preliminary results for 5 human cochlea datasets and could demonstrate that tubes can be selected such that follow-the-leader insertion is achievable. ${ }^{13}$

The contribution of this paper is an extended proof-of-concept study for the use of tubular manipulators as a mean to insert an electrode into the cochlea. We investigate the feasibility of our concept for 22 human cochlea datasets by determining anatomical and material constraints to inform an optimization algorithm. We propose an optimization algorithm which optimizes the tube parameters in order to achieve optimal final positioning within the cochlea and follow-the-leader insertion. We further investigate whether generic tube sets could be used for electrode provision of various cochleas, proving the feasibility of our proposed concept.

\section{TUBULAR MANIPULATORS}

\subsection{Tube Parameters}

The design of tubular manipulators and tube selection is not straight forward, as several tube parameters have to be selected, e.g. curvature, diameter, length. Follow-the-leader behavior of tubular manipulators is possible for certain subsets of precurved tubes. According to Gilbert et al. ${ }^{12}$ tubular manipulators composed of 2 tubes can move in a follow-the-leader fashion, if both tubes have a helical precurvature with equal torsion and an angular displacement between the wire and tube of $n \pi$. As the cochlea exhibits a helical shape, we apply helical precurvatures.

A helical tube is defined by the tube's curvature $u_{x}^{\star}$, torsion $u_{z}^{\star}$, length $l$, the outer $O D$ and inner tube diameter $I D$, the Young's modulus $E$ and the moment of inertia $I$. The helical wire is also defined by the same parameters except the inner tube diameter. The helical radius $r$ and helical pitch $2 \pi p$ of the helical tube and wire are defined by

$$
r=\frac{u_{x}^{\star}}{u_{x}^{\star 2}+u_{z}^{\star 2}}, \quad p=\frac{u_{z}^{\star}}{u_{x}^{\star 2}+u_{z}^{\star 2}} .
$$

We consider the wire and tube made from the shape memory alloy NiTi (nickel and titanium), which sustains strains up to $8 \%$ in its superelastic austenite phase. The wire and tube can be precurved in a heat-treatment process into the desired helical precurvature. 


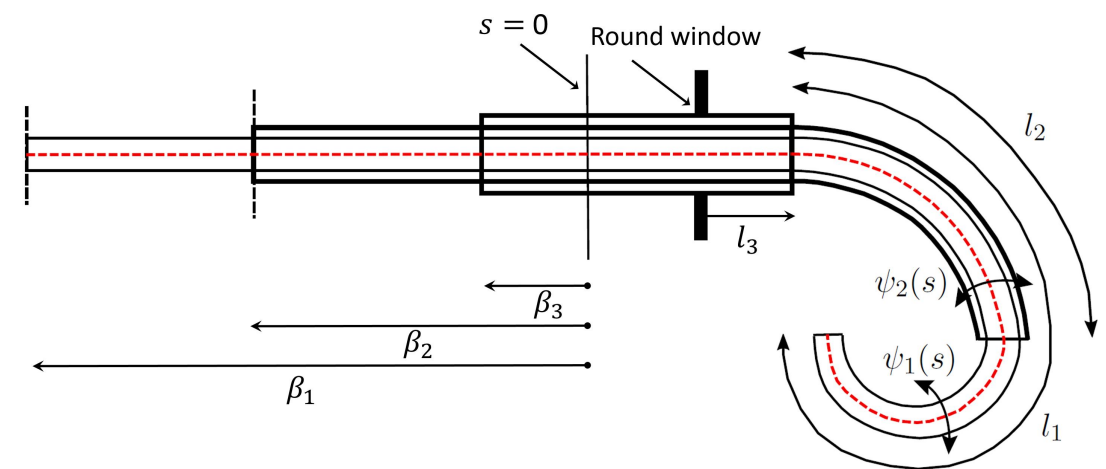

Figure 2: Tubular manipulator with design and actuation parameters and the robot's space curve $g(s)$.

\subsection{Actuation}

Figure 2 illustrates a tubular manipulator composed of the helical shaped inner wire and tube both with straight sections at their base for actuation and a third outer straight tube, which straightens the helical wire and tube for straight delivery to the cochlea. The insertion of the electrode into the cochlea is performed through the round window (RW). Actuation parameters are the lengths $\beta_{1}, \beta_{2}, \beta_{3}$, measured from the constrained outlet $(s=0)$ of the actuation unit to the distal end of the wire and tubes. $l_{1}, l_{2}$ are defined as the lengths from the position where a helical wire or tube leaves the straight tube to the tip of the wire and tube. $l_{3}$ is the insertion depth of the straight tube into the cochlea. $\psi_{1}(s), \psi_{2}(s)$ are the angular displacements of the wire and middle tube respectively.

\subsection{Kinematics}

In this paper, we apply the kinematic model of Rucker et al. ${ }^{14}$ to describe the shape of the manipulator $g(s)$, which accounts for bending and torsion throughout the tubes or wire. To describe the 3D space curve $g(s)$ of the manipulator, parameterized reference frames are established along the curve

$$
g_{i}(s)=\left[\begin{array}{cc}
R_{i}(s) & p_{i}(s) \\
\mathbf{0}^{T} & 1
\end{array}\right]
$$

where $R_{i}(s) \in S O(3)$ denotes the rotation of the frame at arc length $s$, assuming that the z-axis of $R_{i}$ is tangent to the curve and $p_{i}(s) \in \mathbf{R}^{3}$ represents the origin of the frame.

The local curvature vector of tube $i$ is obtained by

$$
\mathbf{u}_{i}=\left(R_{i}^{T} \dot{R}_{i}\right)^{\vee}=R_{1}(s) R_{\Theta_{i}} .
$$

The operator ${ }^{\vee}$ denotes the conversion of a vector in $\mathbf{R}^{3}$ to a skew-symmetric matrix. $R_{\Theta_{i}}$ denotes the rotation about the z-axis of the frame of tube $i$ to tube 1. To describe the precurved shape of the manipulator, FrenetSerret frames are employed as

$$
\mathbf{u}_{i}^{\star}=\left[u_{i x}^{\star}(s) 0 \quad u_{i z}^{\star}(s)\right],
$$

where $u_{i x}^{\star}$ denotes the curvature and $u_{i z}^{\star}$ denotes the torsion of each frame.

The kinematic model applies Kirchhoff rod theory, a special case of Cosserat rod theory, to each component tube as a continuum undergoing bending and torsion. Each tube as a continuum can therefore be expressed by a Kirchhoff beam equation and a set of differential equations is obtained

$$
\begin{aligned}
\dot{\psi}_{i} & =u_{i z}, \\
\dot{u}_{i z} & =\dot{u}_{i z}^{\star}+\frac{1}{E I} \frac{E_{i} I_{i}}{G_{i} J_{i}} \sum_{j=1}^{n} E_{j} I_{j} u_{i x}^{\star} u_{k x}^{\star} \sin \left(\psi_{i}-\psi_{k}\right),
\end{aligned}
$$


where $E I$ denotes the sum over all stiffness, $E I=\sum_{j=1}^{n} E_{j}, I_{j}$ with the stiffness set to zero, if the stiffness of any component tube in the $j t h$ region is not present. $G_{i}$ is the shear modulus and $J_{i}$ the area moment of inertia about the tangent axis.

The boundary conditions for the set of ordinary equations are the axial angles of the component tubes at the constrained outlet

$$
\psi_{i}(0)=\psi_{i 0} \quad i=1 \ldots n
$$

where $\psi_{i}$ is the initial z-axis rotation of component tube $i$ and the vanishing internal moments at the tip

$$
u_{i z}\left(l_{i}\right)=u_{i z}^{\star} \quad i=1 \ldots n .
$$

The relationship of the strains to the internal moment vector is described by a constitutive law

$$
m_{i}=\mathbf{K}_{i}\left(\mathbf{u}_{i}-\mathbf{u}_{i}^{\star}\right),
$$

where $\mathbf{K}$ denotes the stiffness matrix which is dependent on the material properties of the tube, its geometry and the attachment of the reference frame

$$
\mathbf{K}_{i}=\left[\begin{array}{ccc}
k_{1_{i}} & 0 & 0 \\
0 & k_{2_{i}} & 0 \\
0 & 0 & k_{3_{i}}
\end{array}\right]=\left[\begin{array}{ccc}
E_{i} I_{i} & 0 & 0 \\
0 & E_{i} I_{i} & 0 \\
0 & 0 & G_{i} J_{i}
\end{array}\right]
$$

with the Young's modulus $E$ and the constant cross-sectional inertia $I$.

The backbone of the manipulator can then be described by the third curvature component $u_{i z}$ and the local xy-curvature, which is given by

$$
\mathbf{u}_{i x y}=\frac{1}{E I} \sum_{j=1}^{n} E_{j} I_{j} R_{z}\left(\psi_{j}-\psi_{i}\right) \mathbf{u}_{i x y}^{\star}
$$

where $R_{z}$ denotes the rotation about the z-axis. By integrating along the component tubes, one can determine the space curve of the manipulator.

\section{DESIGN CONSIDERATIONS}

\subsection{Anatomical Analysis of the Scala Tympani}

To optimize the helical wire and tube for cochlear electrode insertions, we analyze anatomical shape and size characteristics of 22 scala tympanis segmented from patient computed tomography image data. Design constraints and boundaries are then derived based on the anatomical analysis of the datasets. Segmentation

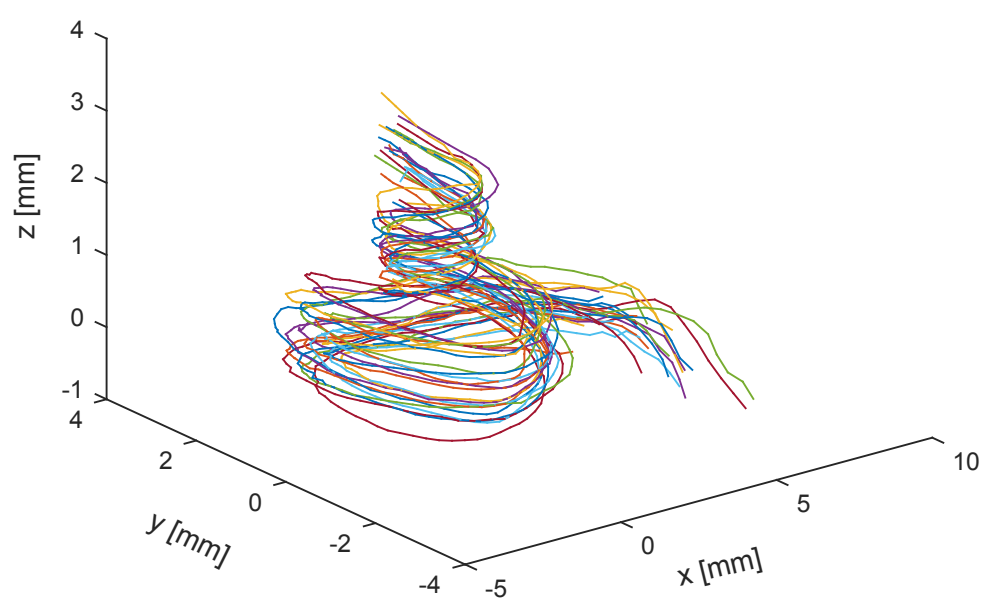

Figure 3: $3 \mathrm{D}$ view of 22 scala tympani centerlines in the consensus coordinate system. was performed using the segmentation algorithm by Noble et al. ${ }^{15}$ The centerlines of the scala tympanis are extracted using an adapted skeletonization algorithm ${ }^{16}$ and transformed into the consensus coordinate system ${ }^{17}$ using Cartesian coordinates for comparison. All centerlines are depicted in Figure 3.

We determine the curvature and mean torsion for each dataset in respect to the length of the scala tympani, which is represented in degrees with $0^{\circ}$ at the intersection of the the $\mathrm{x}$-axis of the consensus coordinate system with the round window, and $800^{\circ}$ corresponding to the apex of the scala tympani. The minimum, maximum, and mean values over all datasets are shown in Figure 4. 

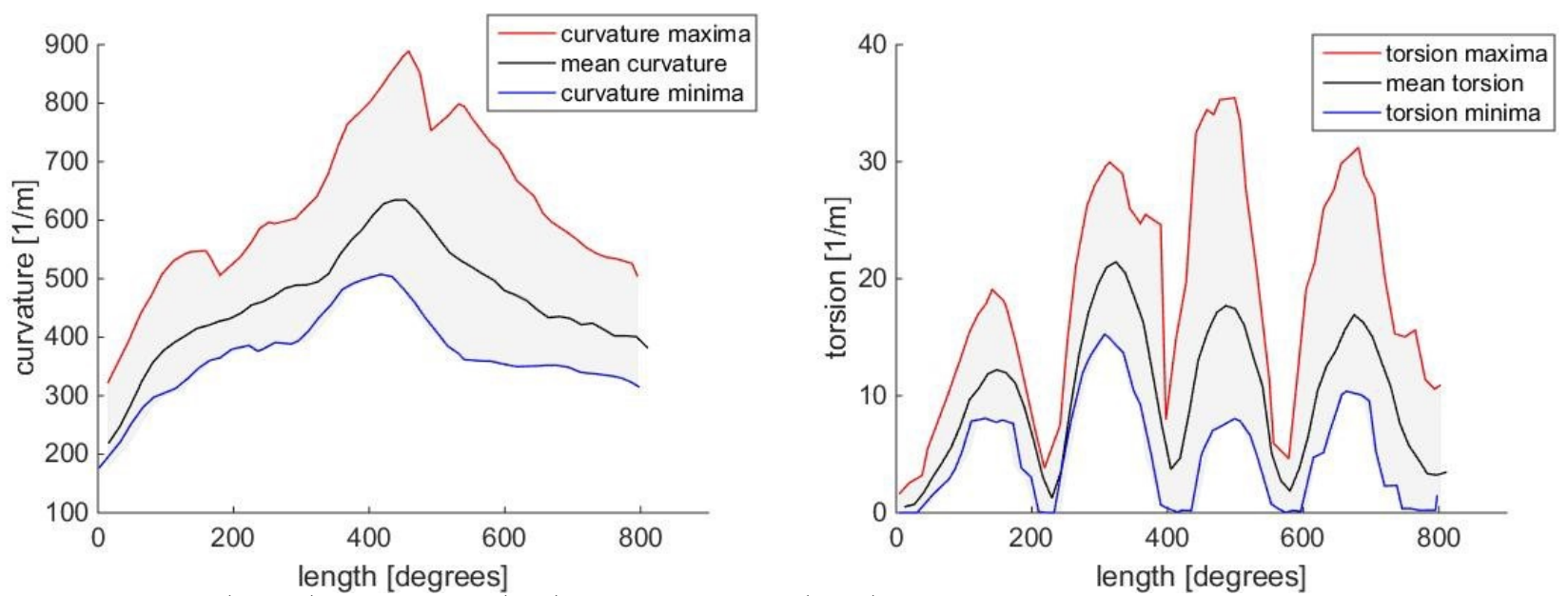

Figure 4: Mean (green), maximum (red), and minimum (blue) curvature and torsion in respect to the length of the scala tympani (in degrees) of 22 scala tympanis.

\subsection{Design Constraints for Helical Tube and Wire}

This contribution focuses on the design of the inner wire, the helical tube and the insertion depth of the straight tube $l_{3}$. To optimize a tubular manipulator for the insertion of a cochlea implant electrode, boundaries for the wire's and tube's diameter, wall thickness and insertion depth are defined. Boundaries for curvatures $u_{1 x}^{\star}$, $u_{2 x}^{\star}$ and torsion $u_{z}^{\star}$ of the helical wire and tube are defined based on the mean values along the centerline for the 22 scala tympanis (Figure 4). NiTi wires can be readily purchased with diameter $D$ as small as $0.08 \mathrm{~mm}$ and NiTi tubes with a wall thickness of $\geq 0.08 \mathrm{~mm}$ (e.g. Euroflex GmbH, Pforzheim, Germany). As the mean canal diameter is $<2 \mathrm{~mm}$ the desired wire's and tube's diameter are set as low as possible. The final insertion depth is defined based on insertion depths of common perimodiolar implants with stylet (Cochlear Ltd., Sydney, Australia and Advanced Bionics LLC, Valencia, USA) and is set to $l_{1}+l_{3}=18 \mathrm{~mm}$.

To ensure a follow-the-leader behavior we define parameter constraints as advised by: ${ }^{12}$ Equal torsion, unequal stiffness and a low curvature ratio of the wire and tube. An angular displacement of $\psi_{2}-\psi_{1}=n \pi$ cannot be guaranteed, as the pitch of the cochlear canal only requires a slight angular displacement between the tube and wire.

\section{TUBULAR MANIPULATOR OPTIMIZATION}

In order to prove whether a tubular manipulator composed of a helical tube and wire can conform to the cochlear shape, we introduce an optimization algorithm. First, we determine if there exists a robot configuration, which is able to conform to the centerline of an individual scala tympani. Second, we evaluate if those tube sets can be actuated such that follow-the-leader motion along the centerline of the scala tympani can be achieved.

\subsection{Optimize Final Position}

For an optimal final position close to the centerline we optimize for design parameters $d=\left[D_{1}, I D_{2}, O D_{2}, l_{1}, l_{2}\right.$, $\left.u_{x}^{*}, u_{z}^{*}, I, E\right]$ and configuration parameters $\left(\psi_{1}, \psi_{2}\right)$ using sequential quadratic programming (SQP). The algorithms determines the wire and tube parameters which minimize an error metric $E M$ by starting at an initial estimate $d_{0}$, meeting prior defined lower $l b$ and upper $u b$ boundaries for $d$ based on anatomical analysis of the scala tympani. To compute the manipulator's space curve for each parameter set, the forward kinematics model is used. $l_{3}$ results from $l_{1}$ and $l_{2}$ to an overall insertion depth of $18 \mathrm{~mm}$. We propose the following error metric to quantify the deviation from the centerline:

$$
E M=\lambda_{1} \frac{\sum_{i}^{n}\|c(i)-p(i)\|_{2}}{n}+\lambda_{2}\|c(n)-p(n)\|_{2},
$$

where $c(i)$ is the arc length $s$ parameterized space curve of the scala tympani's centerline, $p(i)$ is the arc length parameterized space curve of the manipulator, and $s$ is discretized into $n=100$ equidistant points. 


\subsection{Insertion}

To evaluate, whether the tubular manipulator can be actuated in a follow-the-leader motion along the centerline of the scala tympani, we consider the optimized set $d$ from Section 4.1 and define parameters $D_{1}, I D_{2}, O D_{2}, u_{x}^{*}, u_{z}^{*}, I$, $E$ to be constant. For 20 insertion steps into the scala tympani configuration parameters $x_{j}=\left[\beta_{1 j}, \beta_{2 j}, \psi_{1 j}, \psi_{2 j}\right]^{T}$ with $j \in[1,20]$ are determined by minimizing Equation 12 using Nelder-Mead simplex direct search and thus minimizing the deviation of the manipulator's shape from the centerline. $\beta_{3}$ is constant. Both, the manipulator's space curve and part of the centerline for each insertion step are discretized into 100 equidistant points. After simulating the optimal insertion steps for a tube set, the sequence of actuator values is given as $S=\left[x_{1}, \ldots, x_{20}\right]$. To quantify the follow-the-leader error for the insertion, the end-effector position for each tube configuration in $S$ is determined. For each tip position, the Cartesian offset to the space curve of the manipulator's final pose is determined. We define the follow-the-leader error as the maximum Cartesian offset over all 20 configurations.

\subsection{Subset Selection}

For the intended application, it might not be cost efficient to use individually shaped helical tube/wire sets for each patient. Thus, we evaluate the minimum subset over all tube/wire sets determined in Sec. 4.1 which can serve all cochlea datasets. For each of the optimized tube/wire sets we check, how well the manipulator suites the other cochlea datasets for the respective parameters $u_{1 x}^{\star}, u_{2 x}^{\star}, u_{z}^{\star}, E I_{1}$ and $E I_{2}$. To achieve this, we applied the Nelder-Mead simplex direct search method to optimize the final position of the electrode array within the scala tympani using the error metric EM. We parameterize the search as $x_{2}=\left[l_{1}, l_{2}, \psi_{1}, \psi_{2}\right]^{T}$. The tube/wire set, which suites a maximum number of the remaining cochleas with a maximum path accuracy error $<1 \mathrm{~mm}$ is chosen as a subset. Additional subsets are found by selecting the tube/wire sets, which suit the remaining cochleas that could not be provided with the prior chosen subset.

\section{RESULTS}

\subsection{Optimal Final Position}

The helical wire and tube are optimized for each of the 22 scala tympanis for a final position close to the centerline. The initial estimate $d_{0}$ is selected, by generating randomly distributed values considering the constraints for $d$.

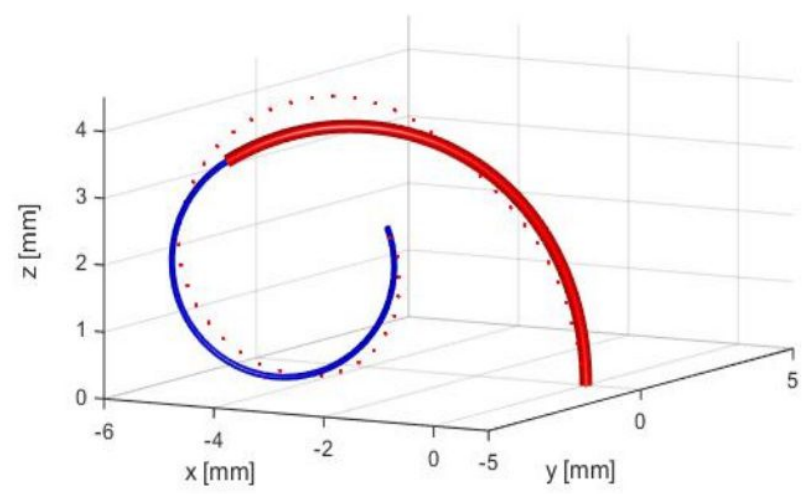

Figure 5: 3D view of final position optimization of the tubular manipulator (red dotted line) with outer tube (red) and inner wire (blue).
To evaluate how well the determined tubular manipulator fits the cochlea's scala tympani centerline, we determine the maximum path accuracy and the tip error. To ensure a save insertion and placement of the array, all values should be $\leq 1 \mathrm{~mm}$. The optimization for 3 cochleas could not be considered successful, as their maximum path accuracy error exceeds $1 \mathrm{~mm}$. Looking at the parameters for those 3 , it is noticeable that the cochleas were the smallest among all datasets such that the centerlines exhibit high curvatures. We note, that the insertion could have been successful if an insertion depth below $18 \mathrm{~mm}$ was chosen. Over all successful 19 datasets the mean maximum path accuracy error was determined as $0.73 \mathrm{~mm}$ and the tip position error as $0.41 \mathrm{~mm}$. Figure 5 illustrates the result for the final position for one example dataset with optimized parameters: $l_{1}=16.3 \mathrm{~mm}$, $l_{2}=8.2 \mathrm{~mm}, D_{1}=0.19 \mathrm{~mm}, I D_{2}=0.29 \mathrm{~mm}, O D_{2}=0.4 \mathrm{~mm}$, $u_{1 x}^{\star}=563 m^{-1}, u_{2 x}^{\star}=52 m^{-1}, u_{z}^{\star}=24 m^{-1}$.

\subsection{Insertion}

For all determined 19 wire/tube sets, we evaluated the insertion process. The initial estimates $x_{0 j}$ are drawn from a random distribution. Each of the 19 electrode insertions for wire/tube sets with a final position close to the centerline was successful. The follow-the-leader error for the overall set is at maximum $0.81 \mathrm{~mm}$. The mean maximum path accuracy error is $0.81 \mathrm{~mm}$ and the mean value for the maximum tip position error for all insertions is $0.64 \mathrm{~mm}$. 


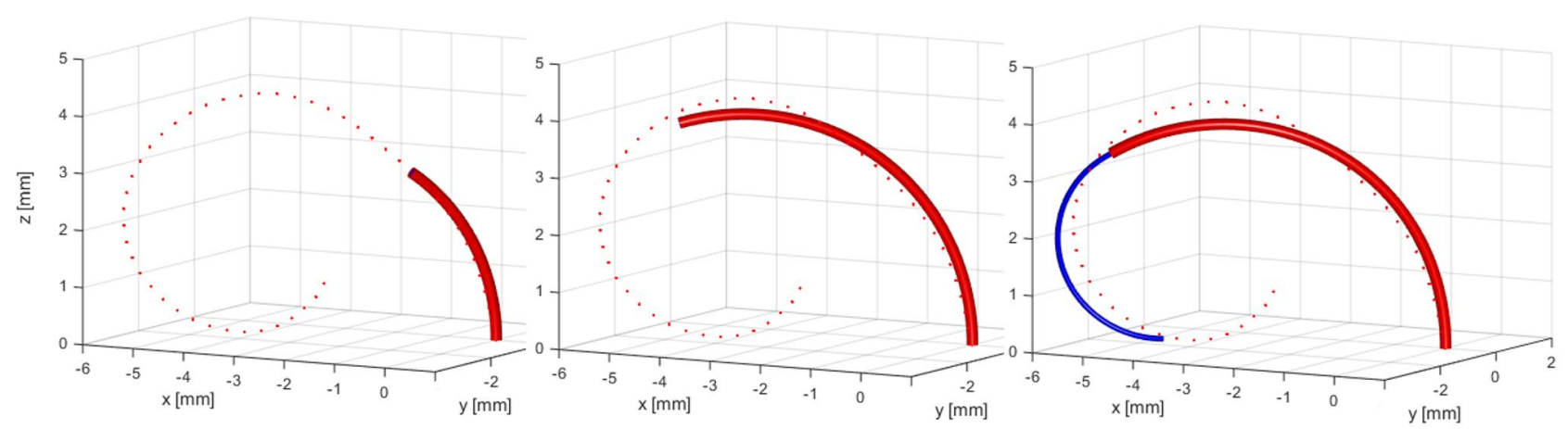

Figure 6: 3 insertion steps for one example dataset with $6.3 \mathrm{~mm}, 10.4 \mathrm{~mm}, 16.1 \mathrm{~mm}$ overall insertion depth.

\subsection{Subsets}

One tube/wire set can provide 8 out of 19 scala tympanis. 7 scala tympanis can be provided with a second tube/wire set, 3 scala tympanis with a third and 1 scala tympani with a fourth tube/wire set. Tube/wire set parameters are summarized in Table 1. This result is promising, as our new steering concept does not require patient-individual tube/wire sets but can be grouped to serve similar cochlea geometries.

Table 1: Minimum subsets over all tube/wire sets $\left(D_{1} / \mathrm{OD}_{2} / \mathrm{ID}_{2}\right.$ in $\mathrm{mm}, u_{i x}^{\star} / u_{z}^{\star}$ in $\mathrm{m}^{-1}, \mathrm{EI}_{i}$ in $\left.\mathrm{GPa} \mathrm{m}^{4}\right)$.

\begin{tabular}{|l|c|c|c|c|c|c|c|c|}
\hline & $\mathrm{D}_{1}$ & $\mathrm{ID}_{2}$ & $\mathrm{OD}_{2}$ & $u_{1 x}^{\star}$ & $u_{2 x}^{\star}$ & $u_{z}^{\star}$ & $E I_{1}$ & $E I_{2}$ \\
\hline Subset 1 & 0.15 & 0.25 & 0.36 & 541.59 & 58.27 & 17.32 & 0.00002 & 0.00002 \\
Subset 2 & 0.23 & 0.33 & 0.44 & 467.33 & 76.33 & 32.20 & 0.00012 & 0.00008 \\
Subset 3 & 0.27 & 0.37 & 0.49 & 409.84 & 42.22 & 15.77 & 0.00021 & 0.00028 \\
Subset 4 & 0.23 & 0.32 & 0.43 & 517.40 & 50.77 & 18.51 & 0.00010 & 0.00008 \\
\hline
\end{tabular}

\section{CONCLUSION}

Developing steerable electrode arrays requires consideration of individual anatomical constraints of the cochlea and careful consideration of the tube parameters of the manipulator. We optimized the tubular manipulator for an optimal final position within the cochlea for 22 datasets. Simulated insertions along the centerline of the scala tympani proved, that follow-the-leader behavior is achievable with maximum path accuracy errors below $0.81 \mathrm{~mm}$. A minimum subset selection revealed, that only 4 tube/wire sets are required to provide 19 scala tympanis. We expect that an extended study of cochlea variances will result in a larger subset of tube/wire combinations, but foresee that those correlate to patient-specific cochlea features and can be hold in stock in a hospital.

Our new concept has the potential to revolutionize the surgical treatment of patients with relevant residual hearing as a contact-less and completely atraumatic insertion becomes feasible by the use of tubular manipulators as a mean for electrode steering. Our next steps consider additional advancements of our optimization algorithm, which should consider the morphology of the cochlea and thus minimize interaction of the electrode with the cochlea walls. Further steps involve the integration of the tubular manipulator with existing insertion tools ${ }^{18-20}$ and building a physical demonstrator. A major challenge is the electrode redesign, as the tube and wire are envisioned to remain within the electrode after insertion.

\section{ACKNOWLEDGMENTS}

This work was funded by the Emmy Noether Programme of the German Research Foundation (DFG) under award number BU 2935/1-1.

\section{REFERENCES}

[1] Pile, J., Cheung, M. Y., Zhang, J., and Simaan, N., "Algorithms and design considerations for robot assisted insertion of perimodiolar electrode arrays," in IEEE International Conference on Robotics and Automation, 2898-2904 (2011). 
[2] Zhang, J. and Simaan, N., "Design of Underactuated Steerable Electrode Arrays for Optimal Insertions," Journal of Mechanisms and Robotics 5(1), 1-11 (2013).

[3] Arcand, B., Shyamsunder, S., and Friedrich, C., "A Fluid Actuator for Thin-Film Electrodes," Journal of Medical Devices 1(70), 70-78 (2007).

[4] Stieghorst, J., Rau, T. S., Majdani, O., Sadowski, G., and Doll, T., "Hydrogel-based self-bending mechanism for cochlear implants," in 13th Int. Conference on Cochlear Implants and Other Implantable Auditory Technologies, 67 (2014).

[5] Min, K. S., Jun, S. B., Lim, Y. S., Park, S. I., and Kim, S. J., "Modiolus-hugging intracochlear electrode array with shape memory alloy," Computational and Mathematical Methods in Medicine 2013, 1-9 (2013).

[6] Chen, B. K., Kha, H. N., and Clark, G. M., "Development of a steerable cochlear implant electrode array," in International Conference on Biomedical Engineering, 15, 607-610 (2007).

[7] Burgner-Kahrs, J., Rucker, D., and Choset, H., "Continuum robots for medical application: A survey," IEEE Transactions on Robotics 31(6), 1261-1280 (2015).

[8] Gosline, A. H., Vasilyev, N. V., Butler, E. J., Folk, C., Cohen, A., Lang, N., Nido, P. J., and Dupont, P. E., "Percutaneous intracardiac beating-heart surgery using metal MEMS tissue approximation tools," International Journal on Robotics Research 31(9), 1081-1093 (2012).

[9] Hendrick, R. J., Herrell, S. D., and Webster III, R. J., "A Multi-Arm Hand-Held Robotic System for Transurethral Laser Prostate Surgery," in IEEE International Conference on Robotics and Automation, $2850-2855$ (2014).

[10] Burgner, J., Rucker, D. C., Gilbert, H. B., Swaney, P. J., Russell, P. T., Weaver, K. D., and Webster III, R. J., "A Telerobotic System for Transnasal Surgery," IEEE/ASME Transactions on Mechatronics 19(3), 996-1006 (2013).

[11] Burgner, J., Swaney, P. J., Lathrop, R. A., Weaver, K. D., and Webster III, R. J., "Debulking from within: A robotic steerable cannula for intracerebral hemorrhage evacuation," IEEE Transactions on Biomedical Engineering 60(9), 2567-2575 (2013).

[12] Gilbert, H. B., Neimat, J., and Webster III, R. J., "Concentric Tube Robots as Steerable Needles : Achieving Follow-the-Leader Deployment," IEEE Transactions on Robotics 31(2), 246-258 (2015).

[13] Rau, T. S., Granna, J., Lenarz, T., Majdani, O., and Burgner-Kahrs, J., "Tubular manipulators: A new concept for intracochlear positioning of an auditory prosthesis," Current Directions in Biomedical Engineering 1, 515-518 (2015).

[14] Rucker, D. C., Webster III, R. J., Chirikjian, G. S., and Cowan, N. J., "Equilibrium Conformations of Concentric-Tube Continuum Robots," International Journal of Robotics Research 29(10), 1263-1280 (2010).

[15] Noble, J. H., Labadie, R. F., Majdani, O., and Dawant, B. M., "Automatic Segmentation of Intra-Cochlear Anatomy in Conventional CR," IEEE Transactions on Biomedical Engineering 58(9), 2625-2632 (2010).

[16] Lee, T., Kashyap, R., and Chu, C., "Building Skeleton Models via 3-D Medial Surface/Axis Thinning Algorithms," Graphical Models and Image Processing 56(6), 462-478 (1994).

[17] Verbist, B. M., Skinner, M. W., Cohen, L. T., Leake, P. A., James, C., Boëx, C., Holden, T. A., Finley, C. C., Roland, P. S., Roland Jr., J. T., Haller, M., Patrick, J. F., Jolly, C. N., Faltys, M. A., Briaire, J. J., and Frijns, J. H., "Consensus panel on a cochlear coordinate system applicable in histologic, physiologic, and radiologic studies of the human cochlea.," Otology $\&$ Neurotology 31(5), 722-730 (2010).

[18] Schurzig, D., Smith, Z. W., Rucker, D. C., Labadie, R. F., and Webster III, R. J., "A Manual Insertion Mechanism for Percutaneous Cochlear Implantation," in Design of Medical Devices Conference, 1-4 (2010).

[19] Kratchman, L. B., Schurzig, D., McRackan, T. R., Balachandran, R., Noble, J. H., Webster III, R. J., and Labadie, R. F., "An automated insertion tool for cochlea implants: another step towards atraumatic cochlear implant surgery," International Journal of Computer Assisted Radiology and Surgery 5(2), 163-171 (2012).

[20] Kobler, J., Beckmann, D., Rau, T. S., Majdani, O., and Ortmaier, T., "An automated insertion tool for cochlear implants with integrated force sensing capability.," International Journal of Computer Assisted Radiology and Surgery 9(3), 481-94 (2014). 